\title{
Compactness issues and bubbling phenomena for the prescribed Gaussian curvature equation on the torus
}

\author{
Luca Galimberti ${ }^{1}$
}

Received: 28 July 2014 / Accepted: 30 April 2015 / Published online: 24 May 2015

(C) Springer-Verlag Berlin Heidelberg 2015

\begin{abstract}
In the spirit of the previous paper (Borer et al., Commun Math Helv, 2015), where we dealt with the case of a closed Riemann surface $\left(M, g_{0}\right)$ of genus greater than one, here we study the behaviour of the conformal metrics $g_{\lambda}$ of prescribed Gauss curvature $K_{g_{\lambda}}=f_{0}+\lambda$ on the torus, when the parameter $\lambda$ tends to one of the boundary points of the interval of existence of $g_{\lambda}$, and we characterize their "bubbling behavior" as in Borer et al. (Commun Math Helv, 2015).
\end{abstract}

Mathematics Subject Classification $53 \mathrm{~A} 30 \cdot 58 \mathrm{E} 30$

\section{Introduction}

Consider a closed, connected Riemann surface $M$, whose Euler characteristic $\chi(M)$ is zero, endowed with a smooth background metric $g_{0}$. In view of the uniformization theorem, it is possible to assume that the Gauss curvature $K_{g_{0}}$ of $g_{0}$ vanishes identically.

The prescribed Gauss curvature equation, which links the curvature of $g_{0}$ to the curvature $K_{g}$ of a conformal metric $g=e^{2 u} g_{0}$, then reads as

$$
K_{g}=-e^{-2 u} \Delta_{g_{0}} u .
$$

Moreover, for convenience, we normalize the volume of $\left(M, g_{0}\right)$ to unity.

Consider a smooth non-constant function $f_{0}: M \rightarrow \mathbb{R}$ with $\max _{p \in M} f_{0}(p)=0$, all of whose maximum points are non-degenerate, and define for $\lambda \in \mathbb{R}$

Communicated by A. Malchiodi.

Supported by SNF Grant 200021_140467/1.

Luca Galimberti

luca.galimberti@math.ethz.ch

1 Departement Mathematik, ETH-Zürich, 8092 Zurich, Switzerland 


$$
f_{\lambda}:=f_{0}+\lambda \text {. }
$$

A natural question is to understand for which values $\lambda$ the function $f_{\lambda}$ is the Gauss curvature of a metric conformal to $g_{0}$. That is equivalent to ask for which values of $\lambda$, the equation

$$
-\Delta_{g_{0}} u=f_{\lambda} e^{2 u}
$$

admits a solution. The paper [3] completely answers the question, giving necessary and sufficient conditions for solving the equation above. More precisely, Eq. (1.1) has a solution if and only if

$$
\int_{M} f_{\lambda} d \mu_{g_{0}}=\int_{M} f_{0} d \mu_{g_{0}}+\lambda<0
$$

and $f_{\lambda}$ is sign changing. (Recall that the volume is equal to one.) Thus, by taking account of the assumptions made on $f_{0}$, we find that Eq. (1.1) is solvable if and only if

$$
0=-\max _{M} f_{0}<\lambda<-\overline{f_{0}}
$$

where $\overline{f_{0}}:=\int_{M} f_{0} d \mu_{g_{0}}$. Set

$$
\Lambda:=\left(0,-\overline{f_{0}}\right), \quad-\overline{f_{0}}:=\lambda_{\max } .
$$

Our goal in this paper is to study the behaviour of the set of solutions of (1.1) when $\lambda$ approches either 0 or $\lambda_{\max }$, a problem left open in [3] and which we solve by means of a blow-up analysis in the spirit of [1]. Our main results are the following:

Theorem 1.1 Let $f_{0} \leq 0$ be a smooth, non-constant function, all of whose maximum points $p_{0}$ are non-degenerate with $f_{0}\left(p_{0}\right)=0$, and for $\lambda \in \mathbb{R}$ let $f_{\lambda}=f_{0}+\lambda$.

Then there exists a sequence $\lambda_{n} \downarrow 0$, a sequence $u_{n}$ of solutions of the equation

$$
-\Delta_{g_{0}} u_{n}=f_{\lambda_{n}} e^{2 u_{n}}
$$

and there exists $I \in \mathbb{N}$ such that, for suitable $p_{n}^{(i)} \rightarrow p_{\infty}^{(i)} \in M$ with $f_{0}\left(p_{\infty}^{(i)}\right)=0,1 \leq i \leq I$, we obtain $u_{n}\left(p_{n}^{(i)}\right) \rightarrow+\infty$ and one of the following:

(i) $u_{n} \rightarrow-\infty$ locally uniformly on compact domains of $M_{\infty}:=M \backslash\left\{p_{\infty}^{(i)} ; 1 \leq i \leq I\right\}$.

(ii) For suitable $r_{n}^{(i)} \downarrow 0$, the following holds:

(a) We have smooth convergence $u_{n} \rightarrow u_{\infty}$ locally on $M_{\infty}$ and $u_{\infty}$ induces a complete metric $g_{\infty}=e^{2 u_{\infty}} g_{0}$ on $M_{\infty}$ of finite total curvature $K_{g_{\infty}}=f_{0}$.

(b) For each $1 \leq i \leq I$, either (1) there holds $r_{n}^{(i)} / \sqrt{\lambda_{n}} \rightarrow 0$ and in local conformal coordinates around $p_{n}^{(i)}$ we have

$$
w_{n}(x):=u_{n}\left(r_{n}^{(i)} x\right)-u_{n}(0)+\log 2 \rightarrow w_{\infty}(x)=\log \left(\frac{2}{1+|x|^{2}}\right)
$$

smoothly locally in $\mathbb{R}^{2}$, where $w_{\infty}$ induces a spherical metric $g_{\infty}=e^{2 w_{\infty}} g_{\mathbb{R}^{2}}$ of curvature $K_{g_{\infty}}=1$ on $\mathbb{R}^{2}$, or (2) we have $r_{n}^{(i)}=\sqrt{\lambda_{n}}$, and in local conformal coordinates around $p_{\infty}^{(i)}$ with a constant $c_{\infty}^{(i)}$ there holds

$$
w_{n}(x)=u_{n}\left(r_{n}^{(i)} x\right)+\log \left(\lambda_{n}\right)+c_{\infty}^{(i)} \rightarrow w_{\infty}(x)
$$

smoothly locally in $\mathbb{R}^{2}$, where the metric $g_{\infty}=e^{2 w_{\infty}} g_{\mathbb{R}^{2}}$ on $\mathbb{R}^{2}$ has finite volume and finite total curvature with $K_{g_{\infty}}(x)=1+(A x, x)$, where $A=\frac{1}{2} \operatorname{Hess}_{f_{0}}\left(p_{\infty}^{(i)}\right)$. 
Moreover, we have

Theorem 1.2 Let $f_{0} \leq 0$ be a smooth, non-constant function. For $\lambda \in \mathbb{R}$ set

$$
\mathcal{C}_{\lambda}:=\left\{u \in H^{1}\left(M ; g_{0}\right): \int_{M} u d \mu_{g_{0}}=0=\int_{M} f_{\lambda} e^{2 u} d \mu_{g_{0}}\right\} .
$$

Then for any arbitrary sequence $\left(\lambda_{n}\right)_{n} \subset \Lambda$ such that $\lambda_{n} \uparrow \lambda_{\max }$ for $n \rightarrow+\infty$, we have that:

(i) there exists a sequence of minimizers $w_{n} \in \mathcal{C}_{\lambda_{n}}$ of the Dirichlet energy such that:

$$
w_{n} \rightarrow 0 \text { in } C^{2, \alpha}(M)
$$

for any $\alpha \in[0,1)$.

(ii) there exists a sequence of solutions $u_{n}$ to equation

$$
-\Delta_{g_{0}} u_{n}=f_{\lambda_{n}} e^{2 u_{n}}
$$

such that $u_{n} \rightarrow-\infty$ uniformly on the whole $M$.

Observation 1.3 We remark that in Theorem 1.2 no assumptions have been made on the nature of the points of maximum of the function $f_{0}$.

Observation 1.4 In contrast to [1], in the present paper the monotonicity of the energy of the solutions $u_{\lambda}$ as a function of $\lambda$ is not obvious. The proof of this fact is perhaps the main new technical achievement in the present work.

\section{Some notation and preliminary results}

In the following section we will recall some well-known results about the existence of solutions to Eq. (1.1) and introduce some notation and concepts used through the rest of the paper. For further details we refer to [3].

For $\lambda \in \mathbb{R}$ consider the set $\mathcal{C}_{\lambda}$ defined by (1.2). Note that for $\lambda \in\left(0,-\min _{M} f_{0}\right)$ the function $f_{\lambda}$ is sign changing and hence $\mathcal{C}_{\lambda} \neq \emptyset$. On the other hand, $\mathcal{C}_{\lambda}=\emptyset$ for $\lambda \leq 0$ or $\lambda \geq-\min _{M} f_{0}$.

The constraints defining $\mathcal{C}_{\lambda}$ are natural; the first allows to apply the direct methods, the second one is motivated by the Gauss-Bonnet theorem.

Lemma 2.1 For $\lambda \in\left(0,-\min _{M} f_{0}\right)$ the $\operatorname{set}_{\lambda}$ is a $C^{\infty}$-Banach manifold.

Proof Define $G^{\lambda}: H^{1}\left(M ; g_{0}\right) \rightarrow \mathbb{R}^{2}$ by letting

$$
G^{\lambda}(u):=\left(\int_{M} u d \mu_{g_{0}}, \int_{M} f_{\lambda} e^{2 u} d \mu_{g_{0}}\right) .
$$

Then $G^{\lambda}$ is smooth and its first derivative is

$$
D G^{\lambda}(u)[v]=\left(\int_{M} v d \mu_{g_{0}}, 2 \int_{M} f_{\lambda} v e^{2 u} d \mu_{g_{0}}\right) .
$$

Notice that $\left(G^{\lambda}\right)^{-1}(0)=\mathcal{C}_{\lambda}$. Pick $u \in \mathcal{C}_{\lambda}$. If we compute $D G^{\lambda}(u)[v]$ with $v \equiv 1$ and then with $v=f_{\lambda}$, we get two vectors of $\mathbb{R}^{2}$ which are linearly independent; therefore $D G^{\lambda}(u)$ is surjective. Since we are in the Hilbert space $H^{1}\left(M ; g_{0}\right)$, we have that it is splitted by the kernel of $D G^{\lambda}(u)$. It follows that $G^{\lambda}$ is a submersion at $u \in \mathcal{C}_{\lambda}$ and then that $\mathcal{C}_{\lambda}$ is a smooth manifold. (For further details we refer to [5].) The lemma is proved. 
In order to find solutions to Eq. (1.1) for $\lambda \in \Lambda$, we minimize the Dirichlet energy $E$

$$
H^{1}\left(M ; g_{0}\right) \ni u \stackrel{E}{\longmapsto} \int_{M}|\nabla u|_{g_{0}}^{2} d \mu_{g_{0}}
$$

in $\mathcal{C}_{\lambda}$. The energy $E$ is coercive on $\mathcal{C}_{\lambda}$ in view of Poincare's inequality and sequentially weakly lower semicontinuous. Furthermore, $\mathcal{C}_{\lambda}$ is weakly sequentially closed as can easily be shown by means of Moser-Trudinger's inequality. Hence the direct method of the calculus of variation applies and for each $\lambda \in \Lambda$ there exists a minimizer $w_{\lambda} \in \mathcal{C}_{\lambda}$.

But in the course of the proof of Lemma 2.1 we have seen that $G^{\lambda}$ is a submersion at any point of $\mathcal{C}_{\lambda}$ : therefore we can apply the Lagrange multipliers rule and obtain

$$
2 \int_{M}\left(\nabla w_{\lambda}, \nabla v\right)_{g_{0}} d \mu_{g_{0}}=\sigma \int_{M} v d \mu_{g_{0}}+2 \mu \int_{M} f_{\lambda} v e^{2 w_{\lambda}} d \mu_{g_{0}}
$$

for every $v \in H^{1}\left(M ; g_{0}\right)$ with suitable $\sigma, \mu \in \mathbb{R}$. Choosing $v \equiv 1$, we obtain

$$
0=\sigma \int_{M} d \mu_{g_{0}}+2 \mu \int_{M} f_{\lambda} e^{2 w_{\lambda}} d \mu_{g_{0}}
$$

hence $\sigma=0$, because $w_{\lambda} \in \mathcal{C}_{\lambda}$. Notice that, by regularity arguments (see [3] for the details), $w_{\lambda} \in C^{\infty}(M)$ and hence $v \equiv e^{-2 w_{\lambda}} \in H^{1}\left(M ; g_{0}\right)$. For this choice of testing function (2.1) gives

$$
0 \geq-2 \int_{M}\left|\nabla w_{\lambda}\right|_{g_{0}}^{2} e^{-2 w_{\lambda}} d \mu_{g_{0}}=\mu \int_{M} f_{\lambda} d \mu_{g_{0}}
$$

If

$$
\int_{M}\left|\nabla w_{\lambda}\right|_{g_{0}}^{2} e^{-2 w_{\lambda}} d \mu_{g_{0}}=0,
$$

we get $w_{\lambda} \equiv$ constant, which is a contradiction, since in $\mathcal{C}_{\lambda}$ there are no constant functions for $\lambda \in \Lambda$. Therefore, since $\int_{M} f_{\lambda} d \mu_{g_{0}}<0$ for $\lambda \in \Lambda$, we obtain

$$
\mu=\mu(\lambda)=-2 \frac{\int_{M}\left|\nabla w_{\lambda}\right|_{g_{0}}^{2} e^{-2 w_{\lambda}} d \mu_{g_{0}}}{\int_{M} f_{\lambda} d \mu_{g_{0}}}>0 .
$$

As a consequence,

$$
u_{\lambda}:=w_{\lambda}+1 / 2 \log \mu(\lambda)
$$

classically solves (1.1).

For the continuation of our analysis and for technical reasons which will become evident later, it is convenient to introduce for $\lambda \in \mathbb{R}$ the set

$$
\mathcal{E}_{\lambda}:=\left\{u \in H^{1}\left(M ; g_{0}\right): 0=\int_{M} f_{\lambda} e^{2 u} d \mu_{g_{0}}\right\},
$$

defined by a single constraint only.

As above, it can be seen that $\mathcal{E}_{\lambda} \neq \varnothing$ if and only if $\lambda \in\left(0,-\min _{M} f_{0}\right)$ and that it is a $C^{\infty}$-Banach manifold.

A priori it is not clear if we may expect that the Dirichlet energy $E$ attains a mimimun in $\mathcal{E}_{\lambda}$; however an elementary argument shows that for $\lambda \in\left(0, \lambda_{\text {max }}\right)$ we have

$$
E\left(u_{\lambda}\right)=\min _{v \in \mathcal{E}_{\lambda}} E(v)=\min _{v \in \mathcal{C}_{\lambda}} E(v)
$$


where $u_{\lambda}$ is defined by (2.2). Indeed, for any $v \in \mathcal{E}_{\lambda}$, we have $v-\bar{v} \in \mathcal{C}_{\lambda}$ and $E(v)=E(v-\bar{v})$, where $\bar{v}:=\int_{M} v d \mu_{g_{0}}$.

Notice finally that for $\lambda=\lambda_{\max }, u \equiv$ constant belongs to $\mathcal{E}_{\lambda}$ and it mimimizes the energy (which is zero). Furthermore, for $\lambda \in\left(\lambda_{\max },-\min _{M} f_{0}\right)$, it is always true that the energy $E$, even though it does not admit a mimimum, is non negative. That suggests to define the following function:

$$
\beta_{\lambda}:= \begin{cases}\int_{M}\left|\nabla u_{\lambda}\right|_{g_{0}}^{2} d \mu_{g_{0}}=E\left(u_{\lambda}\right) & \text { if } \lambda \in\left(0, \lambda_{\max }\right) \\ 0 & \text { if } \lambda \in\left[\lambda_{\max },-\min _{M} f_{0}\right) .\end{cases}
$$

In the next sections, we study the properties of $\beta_{\lambda}$ and use this information to prove, respectively, Theorems 1.1 and 1.2.

\section{Proof of Theorem 1.1}

In this section we will analyse the behaviour of the family $u_{\lambda}$ of solutions to Eq. (1.1) when the parameter $\lambda$ approaches zero and we will prove Theorem 1.1.

The first result is quite elementary but it shows that in an arbitrary neighborhood of zero the function $\beta_{\lambda}$ can achieve arbitrarily large values. More precisely, we can state:

Lemma 3.1 $\lim \sup _{\lambda \downarrow 0, \lambda \in \Lambda} \beta_{\lambda}=+\infty$.

Proof Assume by contradiction that there exists $\delta \in \Lambda$ such that $\sup _{\lambda \in(0, \delta)} \beta_{\lambda}<+\infty$. Choose a sequence $\left(\lambda_{n}\right)_{n} \subset(0, \delta)$ which converges to zero as $n \rightarrow+\infty$. Thus we have $\int_{M}\left|\nabla w_{\lambda_{n}}\right|_{g_{0}}^{2} d \mu_{g_{0}}<+\infty$ uniformly in $n$, where $w_{\lambda_{n}} \in \mathcal{C}_{\lambda_{n}}$ is a minimizer of the energy $E$. Therefore, since the average of $w_{\lambda_{n}}$ is zero, we have, up to subsequences, that $w_{\lambda_{n}} \rightarrow w_{0}$ weakly in $H^{1}\left(M ; g_{0}\right)$ and $e^{2 w_{\lambda_{n}}} \rightarrow e^{2 w_{0}}$ strongly in $L^{1}$. Thus

$$
0=\int_{M} f_{\lambda_{n}} e^{2 w_{\lambda_{n}}} d \mu_{g_{0}} \rightarrow \int_{M} f_{0} e^{2 w_{0}} d \mu_{g_{0}}
$$

and $w_{0} \in \mathcal{E}_{0}=\emptyset$. The contradiction proves the lemma.

In the following, we are going to construct a suitable comparison function belonging to the manifold $\mathcal{E}_{\lambda}$, which will give a control on the rate of blow-up of the mimimum of the energy. This is the content of the next proposition, but before we need:

Lemma 3.2 There exists $L>0$ such that for any $\lambda<-\min _{M} f_{0}$ and for any $p \in M$ point of maximum of $f_{0}$ we have

(i) $\frac{\sqrt{\lambda}}{L}<1$,

(ii) $f_{0}(x)>-\frac{\lambda}{2}$ on $B_{\frac{\sqrt{\lambda}}{L}}(0) \subset \mathbb{R}^{2}$,

where $x$ are suitable local conformal coordinates around $p \simeq 0$.

Proof Fix a point of maximum $p_{i}$ of $f_{0}$. Then, by choosing local conformal coordinates $x$ around $p_{i} \simeq 0$, we have

$$
f_{0}(x)=\frac{1}{2} D^{2} f_{0}(0)[x, x]+O\left(|x|^{3}\right) \text { in } B_{1}(0) \subset \mathbb{R}^{2} .
$$

From the beginning we may assume that $\frac{1}{2} D^{2} f_{0}(0)[x, x] \geq-c_{1}|x|^{2}$, where $c_{1}>0$. Then, for $x \in B_{1}(0)$, we have

$$
f_{0}(x) \geq-c_{1}|x|^{2}-c_{2}|x|^{3} \geq-c\left(|x|^{2}+|x|^{3}\right),
$$

with $c_{2}>0$ and $c:=\max \left(c_{1}, c_{2}\right)>0$. 
Pick $\lambda>0$ and $L_{i}>0$ to be determined later, such that $\sqrt{\lambda} / L_{i}<1$, namely $\lambda<L_{i}^{2}$. Then, on the ball $B_{\frac{\sqrt{\lambda}}{L_{i}}}(0)$ we get

$$
f_{0}(x)>-c\left(\frac{\lambda}{L_{i}^{2}}+\frac{\lambda^{3 / 2}}{L_{i}^{3}}\right) \geq-\frac{\lambda}{2}
$$

where the last inequality holds if we choose $L_{i}^{2} \geq 4 c$. Choose $L_{i}>>0$ so that $-\min _{M} f_{0}<$ $L_{i}^{2}$. Taking $L:=\max _{p_{i}} L_{i}$, we obtain the desired result.

Proposition 3.3 For any $0<\sigma \leq 1$ there exists $\lambda_{\sigma}<1, \lambda_{\sigma} \in \Lambda$, such that for any $0<\lambda \leq \lambda_{\sigma}$ there holds:

$$
\beta_{\lambda} \leq 2 \pi M_{0}(\sigma+2)^{2} \log (1 / \lambda)
$$

where $M_{0}$ is a constant which depends only on $\left(M, g_{0}\right)$ and the function $f_{0}$.

Proof Choose $p_{0} \in M$ such that $f_{0}\left(p_{0}\right)=0$ and choose conformal coordinates $x$ as in the previous Lemma so that

$$
f_{0}(x)+\lambda \geq \frac{\lambda}{2}, \quad x \in B_{\frac{\sqrt{\lambda}}{L}}(0)
$$

for any $\lambda<-\min _{M} f_{0}$. Locally we can write $g_{0}=e^{2 v_{0}} g_{\mathbb{R}^{2}}$ where $v_{0} \in C^{\infty}\left(\overline{B_{1}(0)}\right)$ and $v_{0}(0)=0$. Fix $\lambda \in \Lambda$ with $\lambda<1$. Define the function $\varphi(\lambda): M \rightarrow \mathbb{R}$ as

$$
\varphi(\lambda)(x)= \begin{cases}\log \left(\frac{\sqrt{\lambda}}{L|x|}\right), & \frac{\lambda^{3 / 2}}{L} \leq|x| \leq \frac{\sqrt{\lambda}}{L} \\ \log \left(\frac{1}{\lambda}\right), & |x| \leq \frac{\lambda^{3 / 2}}{L} \\ 0, & \frac{\sqrt{\lambda}}{L} \leq|x| \leq 1\end{cases}
$$

extended to zero on the rest of $M$. We have $\varphi(\lambda) \in H^{1}\left(M ; g_{0}\right)$ and $f_{\lambda}$ is positive on the support of $\varphi(\lambda)$.

Consider the continuous function $z: \mathbb{R} \rightarrow \mathbb{R}$ defined by $z(\alpha)=\int_{M} f_{\lambda} e^{2 \alpha \varphi(\lambda)} d \mu_{g_{0}}$; then $z(0)<0$ and $\lim _{\alpha \rightarrow+\infty} z(\alpha)=+\infty$; thus there exists $\alpha=\alpha(\lambda) \in(0,+\infty)$ where

$$
0=z(\alpha)=\int_{M} f_{\lambda} e^{2 \alpha \varphi(\lambda)} d \mu_{g_{0}},
$$

that is, $\alpha \varphi(\lambda) \in \mathcal{E}_{\lambda}$.

We can give a more precise estimate of $\alpha$, as follows. Recall that $\operatorname{Vol}\left(M ; g_{0}\right)=1$, therefore

$$
\begin{aligned}
0 & =\int_{M} f_{\lambda} e^{2 \alpha \varphi(\lambda)} d \mu_{g_{0}} \geq \lambda / 2 \int_{B_{\frac{\sqrt{\lambda}}{L}}(0)} e^{2 \alpha \varphi(\lambda)} e^{2 v_{0}} d x-\left\|f_{0}\right\|_{\infty} \\
& >\lambda / 2 \int_{B_{\frac{\lambda^{3} / 2}{L}}(0)} e^{2 \alpha \log (1 / \lambda)} e^{2 v_{0}} d x-\left\|f_{0}\right\|_{\infty} .
\end{aligned}
$$

Let $m_{0}:=\min _{B_{1}(0)} e^{2 v_{0}}$ and $M_{0}:=\max _{B_{1}(0)} e^{2 v_{0}}$. We obtain:

$$
\frac{m_{0} \pi}{2} \frac{\lambda^{4-2 \alpha}}{L^{2}} \leq\left\|f_{0}\right\|_{\infty}
$$

or equivalently

$$
0<\alpha \leq \frac{\log \left(\frac{2 L^{2}\left\|f_{0}\right\|_{\infty}}{m_{0} \pi}\right)}{2 \log (1 / \lambda)}+2
$$


Given $0<\sigma \leq 1$, there exists $\lambda_{\sigma}<1, \lambda_{\sigma} \in \Lambda$, such that for any $0<\lambda \leq \lambda_{\sigma}$ we have $\frac{\log \left(\frac{2 L^{2}\left\|f_{0}\right\|_{\infty}}{m_{0} \pi}\right)}{2 \log (1 / \lambda)}<\sigma$. Hence

$$
\alpha^{2} \leq(\sigma+2)^{2}
$$

Next we have:

$$
\int_{M}|\nabla \varphi(\lambda)|_{g_{0}}^{2} d \mu_{g_{0}}=\int_{B_{\frac{\sqrt{\lambda}}{L}}(0) \backslash B_{\frac{\lambda^{3} / 2}{L}}(0)}|x|^{-2} e^{2 v_{0}} d x ;
$$

hence

$$
m_{0} 2 \pi \log (1 / \lambda) \leq \int_{M}|\nabla \varphi(\lambda)|_{g_{0}}^{2} d \mu_{g_{0}} \leq M_{0} 2 \pi \log (1 / \lambda)
$$

We conclude

$$
\beta_{\lambda} \leq \alpha^{2} \int_{M}|\nabla \varphi(\lambda)|_{g_{0}}^{2} d \mu_{g_{0}} \leq 2 \pi M_{0}(\sigma+2)^{2} \log (1 / \lambda),
$$

which proves the proposition.

From Proposition 3.3, by means of elliptic estimates we obtain uniform $L^{\infty}$-bounds for the family $u_{\lambda}$ of solutions of (1.1), away from the boundary of $\Lambda$. More precisely:

Proposition 3.4 Fix $0<\sigma \leq 1$ and let $\lambda_{\sigma}$ be as in Proposition (3.3). Then for any $\lambda^{*} \in$ $\left(0, \lambda_{\sigma}\right)$ we have

$$
\sup _{\lambda^{*} \leq \lambda \leq \lambda_{\sigma}}\left\|u_{\lambda}\right\|_{\infty}<+\infty .
$$

Observation 3.5 Obviously, the estimate above can be improved by replacing the $L^{\infty}$ norm with "higher" norms (use a bootstrap argument), but in the rest of the paper the estimate above will turn out to be sufficient for all our purposes.

Proof of Proposition 3.4 Because of the Sobolev embedding, it is enough to prove that

$$
\sup _{\lambda^{*} \leq \lambda \leq \lambda_{\sigma}}\left\|u_{\lambda}\right\|_{H^{2}}<+\infty
$$

For $\lambda \in\left[\lambda^{*}, \lambda_{\sigma}\right]$ consider the minimizer $w_{\lambda} \in \mathcal{C}_{\lambda}$, which solves the equation

$$
-\Delta_{g_{0}} w_{\lambda}=\mu(\lambda) f_{\lambda} e^{2 w_{\lambda}}
$$

where $\mu(\lambda) \in(0, \infty)$ is a Lagrange multiplier.

From (3.1), we have

$$
\sup _{\lambda^{*} \leq \lambda \leq \lambda_{\sigma}} \beta_{\lambda} \leq C \log \left(1 / \lambda^{*}\right)
$$

Hence, using Poincaré's inequality, we obtain $\left\|w_{\lambda}\right\|_{H^{1}\left(M ; g_{0}\right)}<C$ uniformly in $\lambda$. By the Moser-Trudinger's inequality, for every $p \geq 1$ then there holds:

$$
\sup _{\lambda^{*} \leq \lambda \leq \lambda_{\sigma}} \int_{M} e^{p w_{\lambda}} d \mu_{g_{0}}<C(p)<\infty .
$$

Our claim thus follows once we can give a lower and an upper bound for $\mu(\lambda)$. Inserting $v=f_{\lambda}$ in (2.1), we obtain

$$
\int_{M}\left(\nabla w_{\lambda}, \nabla f_{\lambda}\right)_{g_{0}} d \mu_{g_{0}}=\mu(\lambda) \int_{M}\left(f_{\lambda}\right)^{2} e^{2 w_{\lambda}} d \mu_{g_{0}} .
$$


Since $\lambda_{\sigma}<-\overline{f_{0}}$, we have $0<c \leq\left(\int_{M} f_{\lambda} d \mu_{g_{0}}\right)^{2}$ uniformly in $\lambda \in\left[\lambda^{*}, \lambda_{\sigma}\right]$. Thus, by Hölder

$$
c<\int_{M}\left(f_{\lambda}\right)^{2} e^{2 w_{\lambda}} d \mu_{g_{0}} \int_{M} e^{-2 w_{\lambda}} d \mu_{g_{0}} .
$$

Applying Moser-Trudinger's inequality, we get

$$
c<C \int_{M}\left(f_{\lambda}\right)^{2} e^{2 w_{\lambda}} d \mu_{g_{0}} \exp \left(\frac{1}{4 \pi} \int_{M}\left|\nabla w_{\lambda}\right|_{g_{0}}^{2} d \mu_{g_{0}}\right) .
$$

Thus, we see that $\int_{M}\left(f_{\lambda}\right)^{2} e^{2 w_{\lambda}} d \mu_{g_{0}}$ for $\lambda \in\left[\lambda^{*}, \lambda_{\sigma}\right]$ is uniformly bounded away from zero and, from (3.5), we obtain

$$
\mu(\lambda) \leq C\left(\left\|w_{\lambda}\right\|_{H^{1}\left(M ; g_{0}\right)}\right)<C<\infty
$$

uniformly in $\lambda$.

To see that $\mu(\lambda)$ is also away from zero, we argue by contradiction. Assume that $\inf _{\lambda^{*} \leq \lambda \leq \lambda_{\sigma}} \mu(\lambda)=0$. Take a sequence $\lambda_{n} \in\left[\lambda^{*}, \lambda_{\sigma}\right]$ such that:

$$
\mu\left(\lambda_{n}\right) \rightarrow 0
$$

and $\lambda_{n} \rightarrow \lambda \in\left[\lambda^{*}, \lambda_{\sigma}\right]$. From the estimates above, we can assume, up to subsequences, that $w_{\lambda_{n}} \rightarrow w$ weakly in $H^{1}\left(M ; g_{0}\right)$ and $e^{2 w_{\lambda_{n}}} \rightarrow e^{2 w}$ strongly in $L^{1}$ as $n \rightarrow \infty$. Recall that we have

$$
\int_{M}\left(\nabla w_{\lambda_{n}}, \nabla v\right)_{g_{0}} d \mu_{g_{0}}=\mu\left(\lambda_{n}\right) \int_{M} f_{\lambda_{n}} v e^{2 w_{\lambda_{n}}} d \mu_{g_{0}}
$$

for any $v \in H^{1}\left(M ; g_{0}\right)$. Passing to the limit $n \rightarrow \infty$ in this equation, we obtain

$$
\int_{M}(\nabla w, \nabla v)_{g_{0}} d \mu_{g_{0}}=0
$$

for each $v \in H^{1}\left(M ; g_{0}\right)$, that is $w$ is harmonic. But then $w \equiv 0$ which is clearly impossible. Therefore, we have shown that for $\lambda \in\left[\lambda^{*}, \lambda_{\sigma}\right], \mu(\lambda)$ is uniformly away from 0 and infinity.

In conclusion, we get a uniform bound in $\lambda$ for

$$
\left\|\Delta_{g_{0}} w_{\lambda}\right\|_{L^{2}}=\left\|\mu(\lambda) f_{\lambda} e^{2 w_{\lambda}}\right\|_{L^{2}} .
$$

Hence, by $L^{p}$-elliptic estimates (see for instance [3], p. 24), we have

$$
\left\|w_{\lambda}\right\|_{H^{2}} \leq C\left\{\left\|w_{\lambda}\right\|_{H^{1}}+\left\|\Delta_{g_{0}} w_{\lambda}\right\|_{L^{2}}\right\}<C
$$

uniformly for $\lambda \in\left[\lambda^{*}, \lambda_{\sigma}\right]$. Recalling Eq. (2.2) and the bounds on $\mu(\lambda)$, the bound (3.4) follows.

Remark 3.6 The proposition above is false when $\lambda$ approaches zero. Indeed, an estimate like $\sup _{0<\lambda<\delta} \max _{M} u_{\lambda}<\infty$ for some $\delta$ would lead, in view of Schauder's estimates, to a uniform $C^{2, \alpha}$ bound for $u_{\lambda}$, which clearly contradicts Lemma 3.1.

In the following we show that the function $\beta_{\lambda}$ is monotone decreasing in a suitable right neighborhood of zero, which is crucial for our argument. As a consequence, $\beta_{\lambda}$ will be differentiable almost everywhere. 
Proposition 3.7 There exists $\lambda_{0} \leq \min \left\{1 / 2,-\overline{f_{0}} / 2\right\}$ such that for any $\lambda^{*} \in\left(0, \lambda_{0}\right)$ there exists $\ell\left(\lambda^{*}\right) \in\left(\lambda^{*},-\min _{M} f_{0}\right)$ such that for any $\lambda \in\left(\lambda^{*}, \ell\left(\lambda^{*}\right)\right)$ we have

$$
\beta_{\lambda}<\beta_{\lambda^{*}} .
$$

Furthermore, choosing $\lambda \in\left(0, \lambda_{0}\right), \lambda>\lambda^{*}$, and defining $\ell(\lambda)$ as above, we have $\ell(\lambda)-\lambda \geq$ $\tau=\tau\left(\lambda^{*}\right)>0$ where $\tau$ is a constant not depending on $\lambda$.

Corollary 3.8 There exists $\lambda_{0} \leq \min \left\{1 / 2,-\overline{f_{0}} / 2\right\}$ such that $\beta_{\lambda}$ is strictly monotone decreasing on the interval $\left(0, \lambda_{0}\right)$.

In order to prepare for the proof of Proposition 3.7, define the map $I: H^{1}\left(M ; g_{0}\right) \rightarrow \mathbb{R}$ by letting

$$
I(u):=-\frac{\int_{M} f_{0} e^{2 u} d \mu_{g_{0}}}{\int_{M} e^{2 u} d \mu_{g_{0}}} .
$$

Note that for any $u \in H^{1}\left(M ; g_{0}\right)$ there holds

$$
u \in \mathcal{E}_{I(u)} .
$$

Moreover, we have $I(u) \in\left(0,-\min _{M} f_{0}\right)$ and $I$ is smooth with first derivative given by the following expression:

$$
D I(u)[v]=-2 \frac{\int_{M} f_{I(u)} v e^{2 u} d \mu_{g_{0}}}{\int_{M} e^{2 u} d \mu_{g_{0}}} \quad u, v \in H^{1}\left(M ; g_{0}\right) .
$$

Fix $0<\lambda_{0} \leq \min \left\{1 / 2,-\overline{f_{0}} / 2\right\}$ and for $\lambda^{*} \in\left(0, \lambda_{0}\right)$ let

$$
A\left(\lambda^{*}\right):=\sup _{\lambda^{*} \leq \lambda<\lambda_{0}} \sup _{M} e^{2 u_{\lambda}}
$$

and

$$
a\left(\lambda^{*}\right):=\inf _{\lambda^{*} \leq \lambda<\lambda_{0}} \inf _{M} e^{2 u_{\lambda}} .
$$

Observe that in view of Proposition 3.4, the above functions are well defined if $\lambda_{0}$ is taken small enough, and that $0<a\left(\lambda^{*}\right) \leq A\left(\lambda^{*}\right)<+\infty$. Finally, $A$ is monotone decreasing and $a$ is monotone increasing in $\lambda^{*}$.

We are ready to prove our proposition.

\subsection{Proof of Proposition 3.7}

Proof Fix for convenience $\sigma=1$ and let $\lambda_{\sigma}$ as given in Proposition 3.3. Consider $\min \left\{\lambda_{0}, \lambda_{\sigma}\right\}$, which with a little abuse of notation we will still call $\lambda_{0}$.

We consider $\lambda^{*} \in\left(0, \lambda_{0}\right)$ and $\beta_{\lambda^{*}}=\int_{M}\left|\nabla u^{*}\right|_{g_{0}}^{2} d \mu_{g_{0}}$, where we have used the abbreviation $u^{*} \equiv u_{\lambda^{*}}$. We also set $\varphi^{*} \equiv \varphi\left(\lambda^{*}\right)$, where $\varphi\left(\lambda^{*}\right)$ is the comparison function defined by the Eq. (3.2). (We recall that $\lambda^{*}<\lambda_{0} \leq 1 / 2<1$, therefore $\varphi^{*}$ is well defined.) Thus, we have inequality (3.3) and

$$
\begin{aligned}
\int_{M}\left(\nabla u^{*}, \nabla \varphi^{*}\right)_{g_{0}} d \mu_{g_{0}} & =\int_{M} f_{\lambda * \varphi^{*}} e^{2 u^{*}} d \mu_{g_{0}} \\
& >\frac{\lambda^{*}}{2} \log \left(1 / \lambda^{*}\right) \int_{B_{\frac{\left(\lambda^{*}\right)^{3 / 2}}{L}}(0)} e^{2 u^{*}} e^{2 v_{0}} d x,
\end{aligned}
$$


since $f_{\lambda^{*}} \varphi^{*} \geq 0$ and since $f_{\lambda^{*}} \geq \lambda^{*} / 2$ in the ball $B_{\frac{\sqrt{\lambda^{*}}}{L}}(0)$. Observing that

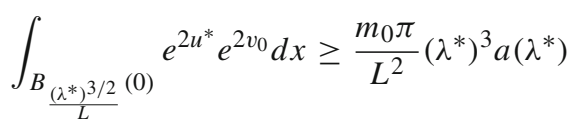

where $a\left(\lambda^{*}\right)$ is defined by (3.10) and $m_{0}=\min _{B_{1}(0)} e^{2 v_{0}}$ as above, we obtain

$$
\begin{aligned}
\int_{M}\left(\nabla u^{*}, \nabla \varphi^{*}\right)_{g_{0}} d \mu_{g_{0}} & =\int_{M} f_{\lambda^{*}} \varphi^{*} e^{2 u^{*}} d \mu_{g_{0}} \\
& >\frac{m_{0} \pi}{2 L^{2}} \log \left(1 / \lambda^{*}\right)\left(\lambda^{*}\right)^{4} a\left(\lambda^{*}\right)>0 .
\end{aligned}
$$

Moreover, using Eqs. (3.1) and (3.3), from Hölder's inequality we deduce

$$
\int_{M}\left(\nabla u^{*}, \nabla \varphi^{*}\right)_{g_{0}} d \mu_{g_{0}} \leq 6 \pi M_{0} \log \left(1 / \lambda^{*}\right)
$$

Hence, defining

$$
\varepsilon^{*}:=2 \frac{\int_{M}\left(\nabla u^{*}, \nabla \varphi^{*}\right)_{g_{0}} d \mu_{g_{0}}}{\int_{M}\left|\nabla \varphi^{*}\right|_{g_{0}}^{2} d \mu_{g_{0}}}
$$

and using inequality (3.12) and once more (3.3), we eventually get

$$
\frac{m_{0}}{2 M_{0} L^{2}}\left(\lambda^{*}\right)^{4} a\left(\lambda^{*}\right)<\varepsilon^{*}<\frac{6 M_{0}}{m_{0}} .
$$

In particular, $\varepsilon^{*}$ is positive. (Recall that $M_{0}:=\max _{B_{1}(0)} e^{2 v_{0}}$ ).

For $\varepsilon \in\left[-\varepsilon^{*}, \varepsilon^{*}\right]$ consider the function $u^{*}-\varepsilon \varphi^{*} \in H^{1}\left(M ; g_{0}\right)$. Recall that by (3.7), we trivially have

$$
u^{*}-\varepsilon \varphi^{*} \in \mathcal{E}_{I\left(u^{*}-\varepsilon \varphi^{*}\right)} .
$$

Lemma 3.9 For $\varepsilon \in\left(0, \varepsilon^{*}\right)$ we have

$$
\beta_{I\left(u^{*}-\varepsilon \varphi^{*}\right)}<\beta_{\lambda^{*}}
$$

Proof By expanding the Dirichlet energy, for $\varepsilon \in\left(0, \varepsilon^{*}\right)$ we obtain

$$
\begin{aligned}
\beta_{I\left(u^{*}-\varepsilon \varphi^{*}\right)} \leq E\left(u^{*}-\varepsilon \varphi^{*}\right) & =E\left(u^{*}\right)-2 \varepsilon \int_{M}\left(\nabla u^{*}, \nabla \varphi^{*}\right)_{g_{0}} d \mu_{g_{0}}+\varepsilon^{2} \int_{M}\left|\nabla \varphi^{*}\right|_{g_{0}}^{2} d \mu_{g_{0}} \\
& =E\left(u^{*}\right)-\varepsilon\left(\varepsilon^{*}-\varepsilon\right) \int_{M}\left|\nabla \varphi^{*}\right|_{g_{0}}^{2} d \mu_{g_{0}} \\
& <E\left(u^{*}\right)=\beta_{\lambda^{*}},
\end{aligned}
$$

as claimed.

The next step is to understand whether the value $I\left(u^{*}-\varepsilon \varphi^{*}\right)$ is greater or smaller than $\lambda^{*}=I\left(u^{*}\right)$. In order to do that, we introduce the function $h:\left[-\varepsilon^{*}, \varepsilon^{*}\right] \rightarrow\left(0,-\min _{M} f_{0}\right)$ given by:

$$
h(\varepsilon):=I\left(u^{*}-\varepsilon \varphi^{*}\right) .
$$

By definition of $I$, we have $h \in C^{1}\left(\left[-\varepsilon^{*}, \varepsilon^{*}\right]\right)$; moreover, there holds: 
Lemma 3.10 We have that

$$
h^{\prime}>0 \text { on }\left[0, \varepsilon^{*}\right] \text {. }
$$

As a consequence, $h$ is smoothly invertible on $\left[0, \varepsilon^{*}\right]$.

Postponing the proof of the lemma, we continue with the proof of Proposition 3.7.

In view of Lemma 3.10, we have $h\left(\varepsilon^{*}\right)>\lambda^{*}$. Furthermore, for any $\lambda \in\left(\lambda^{*}, h\left(\varepsilon^{*}\right)\right)$ there exists a unique $\varepsilon \in\left(0, \varepsilon^{*}\right)$ such that $h(\varepsilon)=I\left(u^{*}-\varepsilon \varphi^{*}\right)=\lambda$. From Lemma 3.9, then we get $\beta_{\lambda}<\beta_{\lambda^{*}}$.

Therefore, setting $\ell\left(\lambda^{*}\right):=h\left(\varepsilon^{*}\right)$, we obtain the first part of Proposition 3.7.

It remains to show the estimate on the length of this interval $\left(\lambda^{*}, \ell\left(\lambda^{*}\right)\right)$ and the relations between it and $(\lambda, \ell(\lambda))$, for $\lambda>\lambda^{*}$. This will be done in Lemma 3.11.

Proof of Lemma 3.10 Recall that $h(0)=\lambda^{*}$. Compute the first derivative of $h$, using (3.8):

$$
h^{\prime}(\varepsilon)=D I\left(u^{*}-\varepsilon \varphi^{*}\right)\left[-\varphi^{*}\right]=2 \frac{\int_{M} f_{I\left(u^{*}-\varepsilon \varphi^{*}\right)} \varphi^{*} e^{2 u^{*}-2 \varepsilon \varphi^{*}} d \mu_{g_{0}}}{\int_{M} e^{2 u^{*}-2 \varepsilon \varphi^{*}} d \mu_{g_{0}}} .
$$

Thus

$$
h^{\prime}(0)=2 \frac{\int_{M} f_{\lambda *} \varphi^{*} e^{2 u^{*}} d \mu_{g_{0}}}{\int_{M} e^{2 u^{*}} d \mu_{g_{0}}}>0
$$

in view of (3.12). By continuity of $h^{\prime}$, there exists $\varepsilon \in\left(0, \varepsilon^{*}\right]$ such that $h^{\prime}>0$ on $[0, \varepsilon)$ and such that $\varepsilon$ is maximal with this property. We claim that $\varepsilon=\varepsilon^{*}$. Suppose by contradiction that $\varepsilon<\varepsilon^{*}$. Note that $h(\varepsilon)=I\left(u^{*}-\varepsilon \varphi^{*}\right)>\lambda^{*}=h(0)$, since $h^{\prime}>0$ on $[0, \varepsilon)$. Moreover,

$$
\begin{aligned}
\int_{M} f_{h(\varepsilon)} \varphi^{*} e^{2 u^{*}-2 \varepsilon \varphi^{*}} d \mu_{g_{0}} & =\int_{B \frac{\sqrt{\lambda^{*}}}{L}} f_{h(\varepsilon)} \varphi^{*} e^{2 u^{*}-2 \varepsilon \varphi^{*}} e^{2 v_{0}} d x \\
& \geq \frac{h(\varepsilon)}{2} \int_{B_{\frac{\sqrt{\lambda^{*}}}{L}}(0)} \varphi^{*} e^{\left(2 u^{*}-2 \varepsilon \varphi^{*}\right)} e^{2 v_{0}} d x
\end{aligned}
$$

where in the last inequality we used the fact that

$$
f_{h(\varepsilon)} \geq \frac{h(\varepsilon)}{2} \text { on } B_{\frac{\sqrt{h(\varepsilon)}}{L}}(0) \supset B_{\frac{\sqrt{\lambda^{*}}}{L}}(0)
$$

(recall Lemma 3.2). Therefore, we obtain

$$
\begin{aligned}
\int_{M} f_{h(\varepsilon)} \varphi^{*} e^{2 u^{*}-2 \varepsilon \varphi^{*}} d \mu_{g_{0}} & \geq \frac{h(\varepsilon)}{2} \log \left(1 / \lambda^{*}\right) \int_{B_{\frac{\left(\lambda^{*}\right)^{3 / 2}}{L}}(0)} e^{\left(2 u^{*}-2 \varepsilon \varphi^{*}\right)} e^{2 v_{0}} d x \\
& >\frac{\lambda^{*}}{2} \log \left(1 / \lambda^{*}\right) \int_{B_{\frac{\left(\lambda^{*}\right)^{3 / 2}}{L}}(0)} e^{\left(2 u^{*}-2 \varepsilon^{*} \varphi^{*}\right)} e^{2 v_{0}} d x \\
& =\frac{\left(\lambda^{*}\right)^{1+2 \epsilon^{*}}}{2} \log \left(1 / \lambda^{*}\right) \int_{B_{\frac{\left(\lambda^{*}\right)^{3 / 2}}{L}}(0)} e^{2 u^{*}} e^{2 v_{0}} d x \\
& \geq \frac{m_{0} \pi}{2 L^{2}} \log \left(1 / \lambda^{*}\right)\left(\lambda^{*}\right)^{4+2 \varepsilon^{*}} a\left(\lambda^{*}\right)>0
\end{aligned}
$$


where in the last line we used (3.11). Thus, we have, since $\varepsilon>0$ and $\varphi^{*} \geq 0$,

$$
\begin{aligned}
h^{\prime}(\varepsilon) & >\frac{m_{0} \pi}{L^{2}} \log \left(1 / \lambda^{*}\right)\left(\lambda^{*}\right)^{4+2 \epsilon^{*}} \frac{a\left(\lambda^{*}\right)}{\int_{M} e^{2 u^{*}-2 \varepsilon \varphi^{*}} d \mu_{g_{0}}} \\
& >\frac{m_{0} \pi}{L^{2}} \log \left(1 / \lambda^{*}\right)\left(\lambda^{*}\right)^{4+2 \epsilon^{*}} \frac{a\left(\lambda^{*}\right)}{\int_{M} e^{2 u^{*}} d \mu_{g_{0}}}>0,
\end{aligned}
$$

contradicting the maximality of $\varepsilon$. Furthermore, reasoning as we have just done, we see that the bound (3.17) holds uniformly on $\left(0, \varepsilon^{*}\right)$. We deduce $h^{\prime}\left(\varepsilon^{*}\right)>0$ and the Lemma is proved.

Lemma 3.11 Let $\lambda_{0}$ be defined as in the proof of Proposition 3.7. Fix $0<\lambda^{*}<\lambda<\lambda_{0}$ and consider $\ell(\lambda)$ given by the first part of Proposition 3.7. Then

$$
\ell(\lambda)-\lambda \geq \tau=\tau\left(\lambda^{*}\right)>0
$$

where $\tau$ is a constant not depending on $\lambda$.

Proof of Lemma 3.11 Let's begin with estimating $\ell\left(\lambda^{*}\right)-\lambda^{*}$. We restart from (3.17), which holds for $\varepsilon \in\left(0, \varepsilon^{*}\right)$. By Eq. (3.14) and by the fact that $\lambda^{*}<1$, we get $\left(\lambda^{*}\right)^{4+2 \epsilon^{*}}>$ $\left(\lambda^{*}\right)^{4+\frac{12 M_{0}}{m_{0}}}$ and $\log \left(1 / \lambda^{*}\right)>\log \left(1 / \lambda_{0}\right)$. Recalling the definition of the auxiliary function $A$ [Eq. (3.9)], we can bound

$$
A\left(\lambda^{*}\right) \geq \int_{M} e^{2 u^{*}} d \mu_{g_{0}}
$$

and obtain

$$
h^{\prime}(\varepsilon)>\frac{m_{0} \pi}{L^{2}} \log \left(1 / \lambda_{0}\right)\left(\lambda^{*}\right)^{4+\frac{12 M_{0}}{m_{0}}} \frac{a\left(\lambda^{*}\right)}{A\left(\lambda^{*}\right)} .
$$

Recalling once more (3.14), with the constant $k_{0}:=\frac{m_{0}^{2} \pi}{2 M_{0} L^{4}} \log \left(1 / \lambda_{0}\right)>0$ we may finally estimate

$$
\begin{aligned}
\ell\left(\lambda^{*}\right)-\lambda^{*}=h\left(\varepsilon^{*}\right)-\lambda^{*} & =\int_{0}^{\varepsilon^{*}} h^{\prime}(\varepsilon) d \varepsilon \\
& >\varepsilon^{*} \frac{m_{0} \pi}{L^{2}} \log \left(1 / \lambda_{0}\right)\left(\lambda^{*}\right)^{4+\frac{12 M_{0}}{m_{0}}} \frac{a\left(\lambda^{*}\right)}{A\left(\lambda^{*}\right)} \\
& >k_{0}\left(\lambda^{*}\right)^{8+\frac{12 M_{0}}{m_{0}}} \frac{\left(a\left(\lambda^{*}\right)\right)^{2}}{A\left(\lambda^{*}\right)},
\end{aligned}
$$

and the function $\left(\lambda^{*}\right)^{8+\frac{12 M_{0}}{m_{0}}} \frac{\left(a\left(\lambda^{*}\right)\right)^{2}}{A\left(\lambda^{*}\right)}$ is not decreasing in $\lambda^{*}$.

Hence, taking $\lambda \in\left(\lambda^{*}, \lambda_{0}\right)$, we deduce

$$
\ell(\lambda)-\lambda>k_{0}(\lambda)^{8+\frac{12 M_{0}}{m_{0}}} \frac{(a(\lambda))^{2}}{A(\lambda)} \geq k_{0}\left(\lambda^{*}\right)^{8+\frac{12 M_{0}}{m_{0}}} \frac{\left(a\left(\lambda^{*}\right)\right)^{2}}{A\left(\lambda^{*}\right)}:=\tau>0 .
$$

The lemma is proved.

This concludes the proof of Proposition 3.7. 


\subsection{A bound for the total curvature}

With the help of Corollary 3.8, Proposition 3.3 and following [4], it is now quite straightforward to show the following estimate for the derivative of $\beta_{\lambda}$ :

Lemma 3.12 There exists a sequence $\left(\lambda_{n}\right)_{n} \subset\left(0, \lambda_{0}\right)$ of points of differentiability for $\beta_{\lambda}$, such that $\lambda_{n} \downarrow 0$ as $n \rightarrow \infty$ and

$$
\left|\beta_{\lambda_{n}}^{\prime}\right| \leq C_{0} / \lambda_{n}
$$

where $C_{0}$ is a positive constant.

Proof By Proposition 3.3, we have $\beta_{\lambda} \leq C \log (1 / \lambda)$, for any $\lambda<\lambda_{0}$. Set $C_{0}:=C+1$ and assume that exists $\tilde{\lambda}<\lambda_{0}$ such that for any $\lambda<\tilde{\lambda}, \lambda$ point of differentiability of $\beta_{\lambda}$, there holds:

$$
\left|\beta_{\lambda}^{\prime}\right|>C_{0} / \lambda
$$

Then we obtain, by Lebesgue's theorem, that

$$
\beta_{\lambda}-\beta_{\tilde{\lambda}} \geq \int_{\lambda}^{\tilde{\lambda}}\left|\beta_{s}^{\prime}\right| d s
$$

and hence

$$
C \log (1 / \lambda) \geq \beta_{\lambda}>\beta_{\tilde{\lambda}}+C_{0} \log (\tilde{\lambda} / \lambda)
$$

Thus, we get

$$
\beta_{\tilde{\lambda}}+C_{0} \log (\tilde{\lambda})-\log (\lambda) \leq 0,
$$

which, for $\lambda$ small enough, is clearly impossible. The lemma is proved.

We can now prove the analogue of Eq. (5.1) in [1]:

Proposition 3.13 Let $\left(\lambda_{n}\right)_{n}$ be a sequence like the one given by Lemma 3.12. and set $u_{n}:=$ $u_{\lambda_{n}}$. Then

$$
\limsup _{n}\left(\lambda_{n} \int_{M} e^{2 u_{n}} d \mu_{g_{0}}\right)<\infty .
$$

Proof Fix $n \in \mathbb{N}$ and set for convenience $\lambda^{*}:=\lambda_{n} \in\left(0, \lambda_{0}\right)$ and $u^{*}:=u_{n}$.

Consider the function $h$ defined by Eq. (3.16), where $\varepsilon^{*}$ and $\varphi^{*}$ are defined as in the proof of Proposition 3.7. For $\lambda_{k} \downarrow \lambda^{*}, \lambda_{k}<h\left(\varepsilon^{*}\right)$, set $\varepsilon_{k}:=h^{-1}\left(\lambda_{k}\right)$. By Lemma 3.10, $\varepsilon_{k} \rightarrow 0$ as $k \rightarrow \infty$. Finally, by Lemma 3.12, we may assume that for all $k$

$$
-\frac{\beta_{\lambda^{*}}-\beta_{\lambda_{k}}}{\lambda^{*}-\lambda_{k}} \leq 2 C_{0} / \lambda^{*}:=C / \lambda^{*}
$$

where $C_{0}$ is the constant of Lemma 3.12.

Observe that $\beta_{\lambda^{*}}-\beta_{\lambda_{k}} \geq E\left(u^{*}\right)-E\left(u^{*}-\varepsilon_{k} \varphi^{*}\right)$, since $u^{*}-\varepsilon_{k} \varphi^{*} \in \mathcal{E}_{I\left(u^{*}-\varepsilon_{k} \varphi^{*}\right)}=\mathcal{E}_{\lambda_{k}}$. Now:

$$
E\left(u^{*}\right)-E\left(u^{*}-\varepsilon_{k} \varphi^{*}\right)=\int_{M}\left(-\varepsilon_{k}^{2}\left|\nabla \varphi^{*}\right|_{g_{0}}^{2}+2 \varepsilon_{k}\left(\nabla u^{*}, \nabla \varphi^{*}\right)_{g_{0}}\right) d \mu_{g_{0}} .
$$

Hence,

$$
\frac{C}{\lambda^{*}} \geq \frac{1}{\lambda_{k}-\lambda^{*}} \int_{M}\left(-\varepsilon_{k}^{2}\left|\nabla \varphi^{*}\right|_{g_{0}}^{2}+2 \varepsilon_{k}\left(\nabla u^{*}, \nabla \varphi^{*}\right)_{g_{0}}\right) d \mu_{g_{0}} .
$$


Recalling that $\varepsilon_{k}=h^{-1}\left(\lambda_{k}\right), h^{-1}\left(\lambda^{*}\right)=0$ and using (3.3), we have

$$
\begin{aligned}
\frac{1}{\lambda_{k}-\lambda^{*}} \int_{M} \varepsilon_{k}^{2}\left|\nabla \varphi^{*}\right|_{g_{0}}^{2} & \leq 2 \pi M_{0} \log \left(1 / \lambda^{*}\right) \frac{h^{-1}\left(\lambda_{k}\right)-h^{-1}\left(\lambda^{*}\right)}{\lambda_{k}-\lambda^{*}} \varepsilon_{k} \\
& \rightarrow 0
\end{aligned}
$$

as $k \rightarrow \infty$, since $h^{-1}$ is differentiable at $\lambda^{*}$ and $\varepsilon_{k}$ goes to zero. Therefore, we may write, with an error term $o(1)$ as $k \rightarrow \infty$, that

$$
\frac{C}{\lambda^{*}} \geq \frac{2 \varepsilon_{k}}{\lambda_{k}-\lambda^{*}} \int_{M}\left(\nabla u^{*}, \nabla \varphi^{*}\right)_{g_{0}} d \mu_{g_{0}}+o(1) .
$$

Thus, when $k \rightarrow \infty$, we obtain

$$
\begin{aligned}
\frac{C}{\lambda^{*}} & \geq 2\left(h^{-1}\right)^{\prime}\left(\lambda^{*}\right) \int_{M}\left(\nabla u^{*}, \nabla \varphi^{*}\right)_{g_{0}} d \mu_{g_{0}} \\
& =\frac{2}{h^{\prime}(0)} \int_{M} f_{\lambda^{*}} \varphi^{*} e^{2 u^{*}} d \mu_{g_{0}} \\
& =\int_{M} e^{2 u^{*}} d \mu_{0}
\end{aligned}
$$

where in the last line we have used the explicit expression of $h^{\prime}(0)$. Going back to the original notation, we have for any $n \in \mathbb{N}$

$$
\int_{M} e^{2 u_{n}} d \mu_{g_{0}} \leq C / \lambda_{n}
$$

which is nothing but Eq. (3.18). The Proposition is proved.

As a consequence of Proposition 3.13 and the Gauss-Bonnet identity $0=\int_{M} f_{\lambda_{n}} e^{2 u_{n}} d \mu_{g_{0}}$, we deduce the uniform bound

$$
\sup _{n \in \mathbb{N}} \int_{M}\left(\left|f_{0}\right|+\lambda_{n}\right) e^{2 u_{n}} d \mu_{g_{0}}<\infty
$$

for the total curvature of $g_{n}=e^{2 u_{n}} g_{0}$.

\subsection{Blow-up analysis}

In this subsection we complete the Proof of Theorem 1.1. For the rest of this part, let $\left(\lambda_{n}\right)_{n}$ be a sequence like the one given by Lemma 3.12 and set $u_{n}:=u_{\lambda_{n}}$. We follow closely Section 5 of [1].

As shown by Ding and Liu [2], we obtain for any open domain $\Omega \subset \subset M^{-}:=$ $\left\{p \in M: f_{0}(p)<0\right\}, \int_{\Omega}\left(\left|\nabla u_{n}^{+}\right|_{g_{0}}^{2}+\left|u_{n}^{+}\right|^{2}\right) d \mu_{g_{0}} \leq C(\Omega)$, where $t^{+}=\max \{t, 0\}, t \in \mathbb{R}$, and hence, as proved in [1], that

$$
u_{n} \leq C^{\prime}(\Omega) .
$$

Thus, if a sequence $\left(u_{n}\right)_{n}$ blows up near a point $p_{0} \in M$ in the sense that for every $r>0$ there holds $\sup _{B_{r}\left(p_{0}\right)} u_{n} \rightarrow+\infty$ (and we know that it is always the case in view of Remark 3.6), necessarily $f_{0}\left(p_{0}\right)=0$. Moreover, there exists a sequence of points $p_{n} \rightarrow p_{0}$ such that for some $r>0, u_{n}\left(p_{n}\right)=\sup _{B_{r}\left(p_{0}\right)} u_{n}$.

Let $p_{0}$ be such a blow-up point for a sequence of solutions $u_{n}$. We introduce local isothermal coordinates $x$ on $B_{r}\left(p_{0}\right)$ around $p_{0}=0$. We can write $g_{0}=e^{2 v_{0}} g_{\mathbb{R}^{2}}$ for some smooth function $v_{0}$. Setting $v_{n}:=u_{n}+v_{0}$, we get

$$
-\Delta v_{n}=\left(f_{0}(x)+\lambda_{n}\right) e^{2 v_{n}} \text { on } B_{R}(0)
$$


for some $R>0$ and there is a sequence $x_{n} \rightarrow 0$ so that

$$
v_{n}\left(x_{n}\right)=\sup _{|x| \leq R} v_{n}(x) \rightarrow+\infty
$$

as $n \rightarrow+\infty$. Moreover, $\Delta v_{n}\left(x_{n}\right) \leq 0$ and thus $f_{0}\left(x_{n}\right)+\lambda_{n} \geq 0$, which leads to

$$
\left|x_{n}\right|^{2} \leq C \lambda_{n}
$$

for some constant $C>0$.

We observe that in the present case we do not have available a uniform global lower bound for the sequence of solutions $u_{n}$ (and hence for $v_{n}$ ) of the kind present in [1]. But we can still show that the analogue of Lemma 5.2 [1] holds true. Indeed, a careful inspection shows that a uniform lower bound is not needed in the proof of Lemma 5.2 [1].

Lemma 3.14 For every $r>0$, there holds

$$
\limsup _{n} \int_{B_{r}(0)}\left(f_{0}+\lambda_{n}\right)^{+} e^{2 v_{n}} d x \geq 2 \pi .
$$

In order to prove Theorem 1.1, as regards part (ii), we would like to imitate the proof of Theorem 1.4 [1]. To do that and to show the convergence results therein, the last ingredient we need is at least a local lower bound for our sequence of solutions $u_{n}$.

The next lemma shows that either the sequence degenerates or that we have a local lower bound. After this Lemma, we will obtain part (i) of Theorem 1.1. To prove part (ii), it will be sufficient to repeat the same reasoning as after Lemma 5.2. in [1].

Lemma 3.15 Let $\left(\lambda_{n}\right)_{n}$ and $\left(u_{n}\right)_{n}$ be defined as above and set

$$
M_{\infty}:=M \backslash\left\{p_{\infty}^{(1)}, \ldots, p_{\infty}^{(I)}\right\}
$$

where $p_{\infty}^{(1)}, \ldots, p_{\infty}^{(I)}$ are blow-up points. Then, up to subsequences, either

(i) $u_{n} \rightarrow-\infty$ locally uniformly on compact domains of $M_{\infty}$, or

(ii) for any compact domain $\Omega \subset \subset M_{\infty}$, there exists a constant $C=C(\Omega) \in \mathbb{R}$ such that

$$
\left.u_{n}\right|_{\Omega}>C(\Omega)
$$

uniformly in $n$.

Proof We fix two open domains $\Omega \subset \subset \tilde{\Omega} \subset \subset M_{\infty}$. From (3.19), for any $n$ we get that $\left.u_{n}\right|_{\tilde{\Omega}} \leq C(\tilde{\Omega})$. We pick an arbitrary point $p \in \bar{\Omega}$ and $r_{p}>0$ so that $B_{r_{p}}(p) \subset \tilde{\Omega}$. If needed, we choose a smaller radius and we consider a conformal chart $\Psi: B_{r_{p}}(p) \rightarrow B_{1}(0) \subset \mathbb{R}^{2}$ with coordinates $x$ so that locally we have $g_{0}=e^{2 v_{0}} g_{\mathbb{R}^{2}}$ with $v_{0} \in C^{\infty}\left(\overline{B_{1}(0)}\right)$. Setting $v_{n}:=u_{n}+v_{0}$, we obtain

$$
-\Delta v_{n}=\left(f_{0}(x)+\lambda_{n}\right) e^{2 v_{n}} \text { on } B_{1}(0) .
$$

Split $v_{n}=v_{n}^{(0)}+v_{n}^{(1)}$, where $v_{n}^{(1)} \in H_{0}^{1}\left(B_{1}(0)\right)$ solves the boundary value problem

$$
\begin{cases}-\Delta v_{n}^{(1)}=\left(f_{0}(x)+\lambda_{n}\right) e^{2 v_{n}} & \text { in } B_{1}(0), \\ v_{n}^{(1)}=0 & \text { on } \partial B_{1}(0)\end{cases}
$$

and $v_{n}^{(0)}$ is harmonic. Hence it follows, uniformly in $n$,

$$
\left\|\Delta v_{n}^{(1)}\right\|_{L^{p}\left(B_{1}(0)\right)} \leq\left\|\Delta v_{n}^{(1)}\right\|_{L^{\infty}\left(B_{1}(0)\right)} \leq C
$$


for any $p \geq 1$. Fixing $p>1$, from elliptic regularity theory we obtain that $\left(v_{n}^{(1)}\right)_{n}$ is bounded in $W^{2, p}\left(B_{1}(0)\right) \hookrightarrow C^{0}\left(\overline{B_{1}(0)}\right)$. From the local upper bound on $\tilde{\Omega}$ for the sequence $\left(u_{n}\right)_{n}$ (and hence for $\left.\left(v_{n}\right)_{n}\right)$, we infer that for any $x \in \overline{B_{1}(0)}$,

$$
v_{n}^{(0)}(x) \leq\left\|v_{n}^{(1)}\right\|_{L^{\infty}\left(B_{1}(0)\right)}+C(\tilde{\Omega}) \leq C
$$

uniformly in $n$. Therefore, Harnack's inequality implies that

$$
\sup _{B_{1 / 2}(0)} v_{n}^{(0)} \leq C_{1} \inf _{B_{1 / 2}(0)} v_{n}^{(0)}+C_{2}
$$

for suitable constants $C_{1}>0$ and $C_{2} \in \mathbb{R}$ depending on $B_{1 / 2}(0)$ but not on $n$.

We see that we have two mutually disjoint cases (up to subsequences):

(i) $\inf _{B_{1 / 2}(0)} v_{n}^{(0)} \rightarrow-\infty$, as $n \rightarrow+\infty$

(ii) $\inf _{B_{1 / 2}(0)} v_{n}^{(0)} \geq-C$, uniformly in $n$.

In the first case, it follows, recalling that $\left(v_{n}^{(1)}\right)_{n}$ is bounded in $L^{\infty}\left(B_{1}(0)\right)$, that

$$
v_{n} \rightarrow-\infty
$$

uniformly in $\overline{B_{1 / 2}(0)}$.

In the second case, we deduce $C<\left.v_{n}\right|_{\overline{B_{1 / 2}(0)}}$ uniformly in $n$.

Since $\bar{\Omega}$ is connected, we conclude that either on $\bar{\Omega}$ the sequence of solutions $u_{n}$ goes uniformly to $-\infty$ or that there exists $C=C(\Omega)$ such that $\left.u_{n}\right|_{\Omega}>C$ for any $n$. The Lemma is proved.

\section{Proof of Theorem 1.2}

In this section, we will analyze the asymptotic behaviour of the family $u_{\lambda}$ of solutions to the prescribed Gaussian curvature equation, when the parameter $\lambda \uparrow-\overline{f_{0}}=\lambda_{\max }$. The main content of this section is the proof of Theorem 1.2.

Proposition 4.1 Let $\beta_{\lambda}$ be defined by Eq. (2.3). Then $\beta_{\lambda} \rightarrow 0$ as $\lambda \uparrow \lambda_{\max }$.

In preparation for the proof of the proposition, consider the Hilbert space $H^{1}\left(M ; g_{0}\right) \times \mathbb{R}$ endowed with the natural scalar product and consider the set

$$
\mathcal{C}:=\left\{(u, \lambda) \in H^{1}\left(M ; g_{0}\right) \times \mathbb{R}: \int_{M} u d \mu_{g_{0}}=0=\int_{M} f_{\lambda} e^{2 u} d \mu_{g_{0}}\right\} .
$$

We claim that $\mathcal{C}$ is a $C^{\infty}$-Banach manifold. Indeed, we define $G: H^{1}\left(M ; g_{0}\right) \times \mathbb{R} \rightarrow \mathbb{R}^{2}$ as:

$$
G(u, \lambda):=\left(\int_{M} u d \mu_{g_{0}} ; \int_{M} f_{\lambda} e^{2 u} d \mu_{g_{0}}\right) .
$$

Then

$$
G^{-1}((0,0))=\mathcal{C}
$$

and $G \in C^{\infty}$ with first Frechet derivative

$$
D G(u, \lambda)[v, t]=\left(\int_{M} v d \mu_{g_{0}} ; 2 \int_{M} f_{\lambda} v e^{2 u} d \mu_{g_{0}}+t \int_{M} e^{2 u} d \mu_{g_{0}}\right)
$$

for any $(v, t) \in H^{1}\left(M ; g_{0}\right) \times \mathbb{R}$. 
For any $(u, \lambda) \in \mathcal{C}$, letting $D G(u, \lambda)$ act on $(1,0)$ and $(0,1)$, we obtain respectively the vectors $(1,0)$ and $\left(0, \int_{M} e^{2 u} d \mu_{g_{0}}\right)$, which are clearly a basis for $\mathbb{R}^{2}$. Moreover, the kernel of $D G(u, \lambda)$ splits $H^{1}\left(M ; g_{0}\right) \times \mathbb{R}$. Thus, $\mathcal{C}$ is a smooth manifold of codimension equal to 2.

Define

$$
\tilde{\mathcal{C}}_{\lambda}:=\mathcal{C} \cap\left\{(w, \mu) \in H^{1}\left(M ; g_{0}\right) \times \mathbb{R}: \mu=\lambda\right\}
$$

that is, the slice of $\mathcal{C}$ determined by the hyperplane in $H^{1}\left(M ; g_{0}\right) \times \mathbb{R}$ of equation $\mu=\lambda$. We observe that this set is not empty for $\lambda \in\left(0,-\min _{M} f_{0}\right)$.

Lemma 4.2 There exist a function $s: \mathcal{C}_{\lambda_{\max }} \rightarrow \mathbb{R}$ and a map $\Theta: \mathcal{C}_{\lambda_{\max }} \times\left(0,-\min _{M} f_{0}\right) \rightarrow$ $H^{1}\left(M ; g_{0}\right)$ such that for any $(u, \lambda) \in \mathcal{C}_{\lambda_{\max }} \times\left(0,-\min _{M} f_{0}\right)$ we have

$$
u+s(u)\left(\lambda-\lambda_{\max }\right)\left(f_{0}-\overline{f_{0}}\right)+\Theta(u, \lambda) \in \mathcal{C}_{\lambda}
$$

and with the property that for any fixed $u \in \mathcal{C}_{\lambda_{\max }}$

$$
\|\Theta(u, \lambda)\|_{H^{1}\left(M ; g_{0}\right)}=o\left(\lambda-\lambda_{\max }\right)
$$

as $\lambda \rightarrow \lambda_{\max }$.

Proof We take $u \in \mathcal{C}_{\lambda_{\text {max }}}, \lambda \in\left(0,-\min _{M} f_{0}\right)$ and consider the vector $\left(s\left(f_{0}-\overline{f_{0}}\right), 1\right) \in$ $H^{1}\left(M ; g_{0}\right) \times \mathbb{R}$ where $s \in \mathbb{R}$. We want to find a suitable $s=s(u)$ such that the vector $\left(s\left(f_{0}-\overline{f_{0}}\right), 1\right)$ belongs to the tangent space $T_{\left(u, \lambda_{\max }\right)} \mathcal{C}$.

That amounts to impose

$$
D G\left(u, \lambda_{\max }\right)\left[s\left(f_{0}-\overline{f_{0}}\right), 1\right]=(0,0)
$$

that is,

$$
\left(\begin{array}{c}
s \int_{M}\left(f_{0}-\overline{f_{0}}\right) d \mu_{g_{0}} \\
2 s \int_{M}\left(f_{0}-\overline{f_{0}}\right)^{2} e^{2 u} d \mu_{g_{0}}+\int_{M} e^{2 u} d \mu_{g_{0}}
\end{array}\right)=\left(\begin{array}{l}
0 \\
0
\end{array}\right)
$$

Since $\int_{M}\left(f_{0}-\overline{f_{0}}\right) d \mu_{g_{0}}=0$, we get from the second equation that

$$
s(u)=-\frac{\int_{M} e^{2 u} d \mu_{g_{0}}}{2 \int_{M}\left(f_{0}-\overline{f_{0}}\right)^{2} e^{2 u} d \mu_{g_{0}}}<0 .
$$

In view of the differentiable structure of $\mathcal{C}$, there exists $\Theta: \mathcal{C}_{\lambda_{\max }} \times\left(0,-\min _{M} f_{0}\right) \rightarrow$ $H^{1}\left(M ; g_{0}\right)$ such that

$$
\left(u+s(u)\left(\lambda-\lambda_{\text {max }}\right)\left(f_{0}-\overline{f_{0}}\right)+\Theta(u, \lambda) ; \lambda\right) \in \tilde{\mathcal{C}_{\lambda}}
$$

and $\|\Theta(u, \lambda)\|_{H^{1}\left(M ; g_{0}\right)}=o\left(\lambda-\lambda_{\max }\right)$ as $\lambda \rightarrow \lambda_{\max }$. The result follows.

Proof of Proposition 4.1 We choose $u \equiv 0 \in \mathcal{C}_{\lambda_{\max }}, \lambda \in\left(0,-\min _{M} f_{0}\right)$ and compute $s$ and $\Theta$ accordingly. Thus, $v_{\lambda}:=s(0)\left(\lambda-\lambda_{\max }\right)\left(f_{0}-\overline{f_{0}}\right)+\Theta(0, \lambda) \in \mathcal{C}_{\lambda}$; we evaluate its $H^{1}\left(M ; g_{0}\right)$ norm

$$
\begin{aligned}
& \left\|s(0)\left(\lambda-\lambda_{\max }\right)\left(f_{0}-\overline{f_{0}}\right)+\Theta(0, \lambda)\right\|_{H^{1}\left(M ; g_{0}\right)} \\
& \quad \leq|s(0)|\left|\lambda-\lambda_{\max }\right|\left\|f_{0}-\overline{f_{0}}\right\|_{H^{1}\left(M ; g_{0}\right)}+o\left(\lambda-\lambda_{\max }\right)
\end{aligned}
$$

and see that it goes to zero as $\lambda \rightarrow \lambda_{\max }$.

Since for $\lambda<\lambda_{\max }$ we have by definition $\beta_{\lambda} \leq E\left(v_{\lambda}\right)$, it follows $\beta_{\lambda} \rightarrow 0$ as $\lambda \uparrow \lambda_{\max }$. 
Proof of Theorem 1.2 (Completed) Let $w_{\lambda} \in \mathcal{C}_{\lambda}$ be a minimizer for $\lambda \in \Lambda$, as the one given in Sect. 2: then, since $\bar{w}_{\lambda}=0$ and $\left\|\nabla w_{\lambda}\right\|_{L^{2}(M)}^{2}=\beta_{\lambda} \rightarrow 0$ when $\lambda \uparrow \lambda_{\max }$, it follows by Poincaré -Wirtinger's inequality that $w_{\lambda} \rightarrow 0$ in $H^{1}\left(M ; g_{0}\right)$.

Applying Moser-Trudinger's inequality, we also have $e^{2 w_{\lambda}} \rightarrow 1$ in $L^{p}(M)$ for any $p \in$ $[1, \infty)$. Therefore, by Hölder's inequality, we obtain that for any $v \in H^{1}\left(M ; g_{0}\right)$

$$
\int_{M} f_{\lambda} v e^{2 w_{\lambda}} d \mu_{g_{0}} \rightarrow \int_{M}\left(f_{0}-\overline{f_{0}}\right) v d \mu_{g_{0}}
$$

when $\lambda \uparrow \lambda_{\max }$. We recall that, for any $\lambda \in \Lambda, w_{\lambda}$ solves

$$
\int_{M}\left(\nabla w_{\lambda}, \nabla v\right)_{g_{0}} d \mu_{g_{0}}=\mu(\lambda) \int_{M} f_{\lambda} v e^{2 w_{\lambda}} d \mu_{g_{0}}, \quad v \in H^{1}\left(M ; g_{0}\right)
$$

where $\mu(\lambda)>0$ is a Lagrange multiplier. Choosing $v=f_{0}-\overline{f_{0}}$, we obtain for $\lambda \uparrow \lambda_{\max }$

$$
0=\lim _{\lambda \uparrow \lambda_{\max }} \mu(\lambda) \int_{M}\left(f_{0}-\overline{f_{0}}\right)^{2} d \mu_{g_{0}}
$$

and therefore $\lim _{\lambda \uparrow \lambda_{\max }} \mu(\lambda)=0$.

Thus, using $L^{p}$-estimates, we obtain

$$
\left\|w_{\lambda}\right\|_{H^{2}(M)} \leq c\left(\left\|\Delta w_{\lambda}\right\|_{L^{2}(M)}+\left\|w_{\lambda}\right\|_{H^{1}(M)}\right) .
$$

Since

$$
\left\|\mu(\lambda) f_{\lambda} e^{2 w_{\lambda}}\right\|_{L^{2}(M)} \leq \mu(\lambda)\left\|f_{\lambda}\right\|_{\infty}\left[\int_{M} e^{4 w_{\lambda}} d \mu_{g_{0}}\right]^{1 / 2}
$$

and $e^{4 w_{\lambda}} \rightarrow 1$ in $L^{1}$ as $\lambda \uparrow \lambda_{\text {max }}$, it follows that $\left\|\Delta w_{\lambda}\right\|_{L^{2}(M)} \rightarrow 0$ and hence $w_{\lambda}$ converges to zero in $H^{2}\left(M, g_{0}\right)$. By Sobolev's embedding results, we also have for any $\alpha \in[0,1)$

$$
w_{\lambda} \rightarrow 0 \text { in } C^{0, \alpha}(M)
$$

when $\lambda \uparrow \lambda_{\max }$.

Thus, using the bootstrap method and Schauder's estimates, we obtain $C^{2, \alpha}$ convergence as well.

Finally, we obtain that

$$
u_{\lambda}:=w_{\lambda}+1 / 2 \log \mu(\lambda),
$$

solution to Eq. (1.1), goes uniformly to $-\infty$ on $M$ when $\lambda \uparrow \lambda_{\max }$ and therefore it can not admit any convergent subsequence.

This concludes the proof of Theorem 1.2.

Remark 4.3 Because of the conformal invariance of the Dirichlet energy and from convergence $\left\|\nabla u_{\lambda}\right\|_{L^{2}(M)}^{2} \rightarrow 0$ as $\lambda \uparrow-\overline{f_{0}}=\lambda_{\max }$, it follows that no "fine structure" can appear in the "limit" geometry of the surfaces $\left(M, e^{2 u_{\lambda}} g_{0}\right)$, independently of how we blow up the scale.

Acknowledgments I would like to thank Michael Struwe for the guidance through the project which led to this paper. 


\section{References}

1. Borer, F., Galimberti, L., Struwe, M.: "Large" conformal metrics of prescribed Gauss curvature on surfaces of higher genus. Comment. Math. Helv. (2015)

2. Ding, W.Y., Liu, J.: A note on the prescribing Gaussian curvature on surfaces. Trans. Am. Math. Soc. 347, 1059-1066 (1995)

3. Kazdan, J.L., Warner, F.W.: Curvature functions for compact 2-manifolds. Ann. Math. 99(2), 14-47 (1974)

4. Struwe, M.: Une estimation asymptotique pour le modèle de Ginzburg-Landau [An asymptotic estimate for the Ginzburg-Landau model]. C. R. Acad. Sci. Paris Sr. I Math. 317(7), 677-680 (1993)

5. Zeidler, E.: Nonlinear Functional Analysis and its Applications III. Springer, New york (1985) 